\title{
Practice of Oral Surgery Post Pandemic (Covid-19) in the Oxford Dental College, Bangalore, India
}

\section{Bhandage $\mathrm{S}^{1}$, Chandhana $\mathrm{AV}^{2 *}$, Kumar $\mathrm{HA}^{3}$ and Daniel $\mathrm{D}^{4}$}

${ }^{1}$ Reader, Department of Oral and Maxillofacial Surgery, Rajiv Gandhi University of Health Sciences, India

${ }^{2}$ Post Graduate Student, Department of Oral and Maxillofacial Surgery, Rajiv Gandhi University of Health Sciences, India

${ }^{3}$ Professor and Head, Department of Oral and Maxillofacial Surgery, Rajiv Gandhi University of Health Sciences, India

${ }^{4}$ Department of Oral and Maxillofacial Surgery, Rajiv Gandhi University of Health Sciences, India

*Corresponding author: Chandhana AV, Department of Oral and Maxillofacial Surgery, The Oxford Dental College, Rajiv Gandhi University of Health Sciences, Bommanahalli, Bangalore, India, Tel: +919741107937; Email: Chandhanaav47@gmail.com

\section{Abstract}

COVID-19 is the currently challenging pandemic situation worldwide. This infection is caused by the severe acute respiratory syndrome corona virus 2.Its spread was rapid initially which has comparatively reduced as of now solely due to practice of guidelines given by WHO and CDC for its control and prevention. Especially practice of maintaining social distance, equipped with mouth mask, regular hand sanitisation, minimising travelling etc. Acceptance of vaccines by the community has improved in facing the present pandemic situation. Most of the population is vaccinated presently in India. This pandemic has impacted worldwide in each aspect and especially greatly in medical field which has led to few modifications in approaching patients and also treatment modifications like addressing emergency situations first. Because of $100 \%$ infection spread rate due to aerosol inducing procedures in dentistry there was a shut in dental treatment for a period of time during the initial times of pandemic except the emergencies. Later with strict precautions and Covid protocols dental treatment has been provided to the community. Tele and virtual consultation has gained a great importance during this pandemic, which most of the doctors and patients have accepted way of addressing the health issues. This paper mentions about change in attitude of patients and practice of oral and maxillofacial surgery in the oxford dental college, Bangalore, Karnataka, India and how in turn this has helped health care system in preventing spread of Covid-19.

Keywords: COVID-19; Infection; Dentistry; Attitude

Abbreviations: BAOMS: British Association of Oral and Maxillofacial Surgeons; CDC: Centers for Disease Control and Prevention; COVID: Coronavirus Disease; NHS: National Health Service; OMFS: Oral and Maxillofacial Surgery; PARA: PPE, Avoid, Restrict, Abbreviate; PPE: Personal Protective Equipment; RT-PCR: Reverse Transcription-Polymerase Chain Reaction; SARS-CoV-2: Severe Acute Respiratory Syndrome Coronavirus 2; WHO: World Health Organization.

\section{Introduction}

Coronavirus disease 2019 (COVID-19), was first detected in Wuhan, China in December, 2019. It is caused by severe acute respiratory syndrome coronavirus 2 (SARS-CoV-2) or COVID-19. It is an emerging infectious disease and a public health problem worldwide. Preventive measures have played an essential role in reducing infection rates and controlling 


\section{Open Access Journal of Dental Sciences}

its spread. Hence the importance of public adherence to the same remains unquestionable during such times. This is in turn influenced by their knowledge, attitude and hygiene practices. Research findings have shown that, coronavirus spreads from human-to-human, mainly through respiratory droplets and contact with contaminated materials, following which touching of face-eye-nose-mouth becomes the major route of entry of the virus. The preliminary symptoms of COVID-19 include fever, cough, fatigue, malaise and shortness of breath. Since there were no guidelines for treatment of Covid-19 or a vaccine, there was initially a complete reliance on public awareness of preventive measures. This played a critical role to minimize its spread. Studies that analysed people's attitude and knowledge on COVID-19 in the form of having higher levels of information on the same, concluded that, both entities were related to more positive attitude and implementation of preventive practices leading to reduction in spread of the epidemic [1].

Hospitals are the primary centres of symptom screening, detection and management in the fight against COVID-19. However, certain factors like crowded populations, close contact between patients and health practitioners and the obligation to be fully operational even during the period of social distancing, increase the risk of disease spreading in these centres [2]. Hence it becomes imperative to focus on raising levels of knowledge, attitude and to promote appropriate practices amongst patients to control the spread of the disease whilst continuing to provide safe and effective health care services to the community [3]. This will also in turnaid hospital managers and national governments during implementation of municipal policies in fight against COVID-19. Official sources for COVID-19 information that are hourly updated by international organizations like CDC,WHO, through their websites are actively strengthening the community by educating about the pandemic through many preferred social media platforms like Facebook, Instagram, YouTube etc. This has played a key role in improving hygiene practices in populations. Hospitals have been implementing many rigorous pandemic control and preventive measures and policies like suspension of inpatient visiting, mandatory medical status declaration and face-covering, ample hand hygiene kits, educational information, social distancing rule and warnings broadcasting within hospital locations. Repeated exposure to the hospital's mandatory protocol system, during their frequent visits for examination and treatment, strengthen their good practices in control and prevention of the disease. More frequent the hospital visits, the higher the possibility of good practices [2].

Dental services due to their unique nature like generation of aerosol, sharps handling and health worker's closeness to the oropharyngeal region of the patient contributes to the highest risk of contracting and transmitting the Covid infection [4]. Also there are no specific anti-viral therapeutic agents for the treatment and prevention of COVID-19 [5]. This necessitates the significance of mandating the precautionary measures for Covid infection.

Guidance from the British Association of Oral and Maxillofacial Surgeons (BAOMS) and ENT UK supported the use of telephone and video consultation triage as part of a "PARA" approach (PPE, Avoid, Restrict, Abbreviate) [6]. The NHS has recognised the huge increase in tele consultations across all specialties of medicine as a result of COVID-19 [7]. Tele dentistry and virtual visits, post-pandemic has gained more importance post pandemic. Initially when many private practitioners were sceptical about going back to practising after the lock down period, the philosophy of 'best practice dentistry' helped many to go back to or continue practising, which is nothing but the "best way" to identify, collect, evaluate, disseminate and implement information and monitor outcomes of dental interventions for patients/ population groups [8].

\section{COVID 19 and OMFS}

In fight against infectious diseases, knowledge and appropriate attitudes of students regarding infectious diseases, especially pandemics should be developed during their preclinical study [9]. Clinicians should be wearing disposable gowns, face shields, face masks, gloves and any new Occupational Safety and Health Administration (OSHA) PPE recommendations that will add on to existing guidelines [8].

As in every medical field, oral and maxillofacial surgery is also following Covid protocol while encountering the patients. Being a resident in the department of oral and maxillofacial surgery in this institute, there were few drastic changes noted during the pandemic, which are enumerated. Patient inflow has reduced considerably since the onset of pandemic. Since mid of the year 2021, most patients who walk in are vaccinated for Covid infection, although mostly with first dose only. Patients are examined only if they have a recent Covid test (RT PCR) report except in cases of emergencies like trauma, abscess etc. Patients without a recent Covid test report are advised to get the same and if they complain of pain, they have been advised analgesics for about 3 days. In the institute we have maintained a man handled kiosk at the reception since the time of pandemic comprising of a resident and a specialist doctor who are responsible for taking a brief history of the patient, hereby reducing risk load in terms of Covid-19 spread.

In the beginning of the pandemic, in the year 2020, patients were asked to rinse their mouth with hydrogen peroxide mouthwash before the examination. Patients with 
a negative Covid report were treated on the same day of diagnosis if required or were given appointments just like in pre pandemic era without discrimination. Major cases like fracture or any pathology were treated under general anaesthesia only after being tested Covid negative.

\section{Attitude of Patients}

Some of the observations noted since the time of Covid outbreak saw a change through the pandemic.

Encouraging patients to wear two layered cloth pieces around their face if they couldn't afford an N 95 mask proved to be helpful in the initial stage of the pandemic. Initially, patients had to be reminded to wear mouth masks but presently they are aware about the same. Post pandemic there has been a stark decrease in patient inflow hinting at negligence of dental health.

Initially when the COVID-19 pandemic struck, patients were advised for Covid tests, not all patients complied, however, presently all the patients are readily agreeing with the institution protocol, for instance Covid report in the institution is considered as valid only for 7 days, and if patient has to get treatment after 7 days of Covid test, then they have to go through a test again and all patients are complying with the rule. This helps reinstill the importance of Covid test amongst patients. Certain measures like having a free government aided testing booth right at the entrance of the institute have encouraged patients positively. It is noted now that patients are sanitising their hands with the sanitizer installed at the entrance of the department, without anyone's instructions. After the pandemic the cost of treatments has increased in the institution and this hasn't discouraged patients from undergoing treatment.

\section{Attitude of Doctors (Oral Surgeons)}

All the doctors at the institute have been following the universal hygiene protocol in the pre pandemic area which only has been accessorised after the pandemic.

All the doctors are trained to educate and create awareness amongst patients during this pandemic about infection routes and the importance of getting Covid RT-PCR tests before every appointment and also the importance of getting vaccinated. In the study conducted in Libyan population, participants agreed on the fact that vaccination reduces morbidity and mortality [10].

The following are the precautionary measures followed in oral surgery department of our institute.

- All the doctors are using different mouth masks inside and outside the clinics (working area).
- Doctors are disposing their used mouth masks immediately when they are leaving the working area.

- All the aerosol inducing procedures are carried out with a face shield and PPE (personal protective equipment) kit without failure.

- To reduce the viral (microbial) load in the oral cavity few doctors were using viro-shield mouth spray initially, presently its use has been reduced amongst the doctors in the institution.

- Initially during the pandemic all the OP (out-patient) cards of patients were kept in the institution only - to prevent spread of infection, but now OP cards are given back to the respective patients.

- Disinfection of rooms and equipments with fumigation and cleaning with $1 \%$ sodium hypochlorite as suggested by WHO has been the norm since the time of the pandemic.

\section{Conclusion}

We conclude here with an affirmation on the importance of implementing precautionary measures at every step in health care system to face the challenges of the COVID- 19 pandemic, as this has helped us as a department to control spread of the disease and avoid casualties.

\section{References}

1. Nguyen HB, Nguyen TH, Tran TT, Vo TH, Tran VH, et al. (2021) Knowledge, Attitudes, Practices, and Related Factors Towards COVID-19 Prevention Among Patients at University Medical Center Ho Chi Minh City, Vietnam. Risk Manag Healthc Policy 14: 2119-2132.

2. Feleke BT, Wale MZ, Yirsaw MT (2021) Knowledge, attitude and preventive practice towards COVID-19 and associated factors among outpatient service visitors at Debre Markos compressive specialized hospital, northwest Ethiopia, 2020. Plos one 16(7): e0251708.

3. Al Hanawi MK, Angawi K, Alshareef N, Qattan A, Helmy HZ, et al. (2020) Knowledge, attitude and practice toward COVID-19 among the public in the Kingdom of Saudi Arabia: a cross-sectional study. Frontiers in public health 8: 217.

4. Singh KT, Mishra G, Shukla AK, Behera S, Tiwari AK, et al. (2020) Preparedness among dental professionals towards COVID-19 in India. Pan Afr Medical Journal 36.

5. Muralidar S, Ambi SV, Sekaran S, Krishnan UM (2020) The emergence of COVID-19 as a global pandemic: Understanding the epidemiology, immune response and potential therapeutic targets of SARS-CoV-2. Biochimie 179: 85-100. 
6. Al Izzi T, Breeze J, Elledge R (2020) Following COVID-19 clinicians now overwhelmingly accept virtual clinics in Oral and Maxillofacial Surgery. British Journal of Oral and Maxillofacial Surgery 58(10): 290-295.

7. Horgan TJ, Alsabbagh AY, McGoldrick DM, Bhatia SK, Messahel A (2021) Oral and maxillofacial surgery patient satisfaction with telephone consultations during the COVID-19 pandemic. Br J Oral Maxillofac Surg 59(3): 335-340.

8. Krishnan V (2020) Coping with COVID-19-The life changing pandemic. J World Fed Orthod 9(2): 45-46.

9. Ataş 0, Yildirim TT (2020) Evaluation of knowledge, attitudes, and clinical education of dental students about COVID-19 pandemic. Peer J 8: e9575.

10. Elhadi M, Alsoufi A, Alhadi A, Hmeida A, Alshareea E, et al. (2021) Knowledge, attitude, and acceptance of healthcare workers and the public regarding the COVID-19 vaccine: a cross-sectional study. BMC Public Health 21(1): 1-21. 